and young project members are contributing to the write up of the project findings.

Results The final questionnaires are a true reflection of what the young participants feel are relevant issues to explore with young patients moving from paediatric to adult care. 596 questionnaires were completed by patients during the project data collection phase, demonstrating that the questionnaires were acceptable to the target population. Of these, 115 were delivered by the young person's advisory group members who gave over 100 hours of their summer holiday to take part in the project.

Conclusion The importance of co-production of healthcare research and quality improvement is gaining recognition but examples in paediatric practice are still rare. To our knowledge this is the largest co-produced patient experience project into paediatric transition. The success of the delivery of the project is grounded in true co-production throughout the project. Novel participatory strategies and a level playing field for all project members are key aspects of this work.

\section{G546(P) ABSTRACT WITHDRAWN}

\section{G547(P) THE EFFECT OF HAVING TRANSITION DISCUSSIONS OF PAEDIATRIC PATIENTS; A CO-PRODUCED PATIENT EXPERIENCE STUDY}

${ }^{1} \mathrm{R}$ Bain, ${ }^{2,3} \mathrm{~N}$ Davidson, ${ }^{2,3} \mathrm{~J}$ Ball, ${ }^{3}$ Members of YPAGne, ${ }^{1} \mathrm{~A}$ Wallace, ${ }^{2} \mathrm{~A}$ Bowey, A Battersby. ${ }^{1}$ Faculty of Medical Sciences, Newcastle University, Newcastle upon Tyne, UK; ${ }^{2}$ Great North Children's Hospital, Newcastle upon Tyne Hospitals NHS FT, Newcastle upon Tyne, UK; ${ }^{3}$ Young Person's Advisory Group North England, Newcastle upon Tyne Hospitals NHS FT, Newcastle upon Tyne, UK

\subsection{6/archdischild-2020-rcpch.464}

Aims NICE guidelines (NG43) recommend that discussions between patients and clinicians about transition from paediatric to adult health care should occur by the ages of 1314 years to facilitate a gradual transition into adult healthcare. However, often these conversations are not part of routine consultations at this age. We aimed to evaluate the benefit and importance of having transition discussions and how having them early can impact on patients and their carers.

Methods A survey about patient experience was co-designed with young people. The survey was delivered by researchers and young people in all outpatient departments in a tertiary paediatric hospital. Patients and their carers were asked to complete a survey about their experience and understanding of discussions about transition. They were asked to complete the questionnaires separately. A separate questionnaire was devised for children and young people with learning difficulties.

Results 556 patients and 271 carers completed the survey with sufficient data to be analysed. Only $30.1 \%$ of patients had received a transition discussion by the age of 18 and only $3.4 \%$ of patients received a discussion about transition by the age of 14 . The mean age of transition discussions was 15.8 yrs. Of patients who had received a discussion on transition, there was an improved overall care experience (mean increase of 9.1\%, $\mathrm{p}=0.003$ ) and an overall improvement in patients' perceived preparedness for transition (mean increase of $20.2 \%, \mathrm{p}<0.0001)$. Of carers whose children had discussed transition, they were less anxious (mean decrease of $20.1 \%$, $\mathrm{p}=0.0001)$.

Conclusions Our study suggests that having transition discussions with patients helps to make them feel more prepared for the transition into adult healthcare and reduces carer anxiety relating to transition. There is also an overall improvement in patient experience. This implies that the discussions that are being had are effective in informing patients of the process however, more of these discussions need to be had to empower patients during consultations. Further work is needed to see if there is correlation with improved patient experience and healthcare outcomes.

\section{G548(P) WHAT DO YOUNG PEOPLE THINK ARE THE FACTORS LEADING TO MEDICATION NONADHERENCE, AND HOW CAN THIS INFORMATION BE USED TO IMPROVE SERVICES?}

${ }^{1,2} \mathrm{EJ}$ Farr, ${ }^{3} \mathrm{SMA}$ Alam, ${ }^{1} \mathrm{~S}$ Thomas, ${ }^{1,2} \mathrm{JH}$ Van der Voort. 'Children's Kidney Centre, University Hospital of Wales, Cardiff, UK; ${ }^{2}$ School of Paediatrics, Health Education and Improvement Wales, Cardiff, UK; ${ }^{3}$ Paediatric Nephrology, Birmingham Children's Hospital, Birmingham, UK

\subsection{6/archdischild-2020-rcpch.465}

Aims Those who receive kidney transplants in adolescence and young adulthood have shorter graft survival than those receiving grafts earlier or later in life. Graft loss is multifactorial, but treatment nonadherence and transition from paediatric to adult services have been identified as contributors. The perspectives of young people (YP) regarding medication nonadherence were investigated in a qualitative service improvement project aiming to improve graft health and the experiences of YP transitioning from a tertiary paediatric nephrology service to adult services.

Methods Institutional ethics approval was sought but deemed unnecessary and informed written consent was gained. Young people attending a renal patient summer camp were questioned in two semi-structured focus group discussions ran in parallel by two researchers. Transcribed responses were thematically analysed by two investigators independently, then agreed upon jointly.

Results There were 11 participants (13-30 y, average $21 \mathrm{y}$, $36 \%$ female). Five themes emerged: 1) Staff approach, either positive or negative; 2) Challenging life events; 3) Peer support; 4) Chances to talk; and 5) Patient-related factors. Patient-related factors subdivided into exercising autonomy, no perceived immediate consequences, and normalising \& denial.

Conclusions Healthcare services and consultations involving young people should incorporate their own suggestions to help guard against nonadherence. Young people wanted staff to value and support them. Being scolded by staff when revealing nonadherence led to disengagement, and threats about consequences were counterproductive. YP suggested a more appropriate response would be to work through reasons behind nonadherence. Given that YP reported that nonadherence can be a form of choice and control, ensuring that YP feel involved with decision making and can exercise autonomy in other ways may address this. 